\title{
NUCLEAR PSEUDO-DIFFERENTIAL OPERATORS IN BESOV SPACES ON COMPACT LIE GROUPS
}

\author{
DUVÁN CARDONA
}

\begin{abstract}
In this work we establish the metric approximation property for Besov spaces defined on arbitrary compact Lie groups. As a consequence of this fact, we investigate trace formulae for nuclear Fourier multipliers on Besov spaces. Finally, we study the $r$-nuclearity, the Grothendieck-Lidskii formula and the (nuclear) trace of pseudo-differential operators in generalized Hörmander classes acting on periodic Besov spaces. We will restrict our attention to pseudo-differential operators with symbols of limited regularity.

MSC 2010. Primary 47B10, 46B28; Secondary 22E30, 47G30.
\end{abstract}

\section{INTRODUCTION}

It was reported by H. Feichtinger, H. Führ and I. Pesenson in [21] (see also references therein) that there exist many real life problems in signal analysis and information theory which would require non-euclidean models. These models include: spheres, projective spaces and general compact manifolds, hyperboloids and general non-compact symmetric spaces, and finally various Lie groups. In connection with these spaces it is important to study approximation theory, spacefrequency localized frames, and Besov spaces, on compact and non-compact manifolds. Motivated by these facts, in this paper we prove the approximation property of Grothendieck for Besov spaces defined on general compact Lie groups. This property is of geometric nature and has important consequences in the theory of nuclear operators on Banach spaces [22]. Consequently, by using the aproximation property on Besov spaces we investigate the $r$-nuclearity of global pseudo-differential operators on such spaces. This is possible if we take under consideration the formulation of Besov spaces reported by E. Nursultanov, M. Ruzhansky and M. Tikhonov in [38], in the context of matrix-valued (or full) symbols of global pseudo-differential operators developed by M. Ruzhansky and V. Turunen in terms of the representation theory of compact Lie groups [40].

In order to formulate our work we precise some definitions as follows. Through the work of A. Grothendieck and others methods in spectral theory, the theory of nuclear operators on Banach spaces has attracted much interest in the literature during the last fifty years, due to its applications in the problem of distribution

Key words and phrases. Besov spaces, nuclear trace, pseudo-differential operator, compact Lie group, approximation property.

The author was supported by the Faculty of Sciences of the Universidad de los Andes, Project: Operadores en grupos de Lie compactos, 2016-I. No new data was created or generated during the course of this research. 
of eigenvalues. Let us consider $E$ and $F$ be two Banach spaces and let $0<r \leq 1$. Following A. Grothendieck [22], Chapter II, p. 3, a linear operator $T: E \rightarrow F$ is $r$-nuclear, if there exist sequences $\left(e_{n}^{\prime}\right)_{n}$ in $E^{\prime}$ (the dual space of $E$ ) and $\left(y_{n}\right)_{n}$ in $F$ such that

$$
T f=\sum_{n} e_{n}^{\prime}(f) y_{n}
$$

and

$$
\sum_{n}\left\|e_{n}^{\prime}\right\|_{E^{\prime}}^{r}\left\|y_{n}\right\|_{F}^{r}<\infty .
$$

The class of $r$-nuclear operators is usually endowed with the quasi-norm

$$
n_{r}(T):=\inf \left\{\left\{\sum_{n}\left\|e_{n}^{\prime}\right\|_{E^{\prime}}^{r}\left\|y_{n}\right\|_{F}^{r}\right\}^{\frac{1}{r}}: T=\sum_{n} e_{n}^{\prime} \otimes y_{n}\right\}
$$

and, if $r=1, n_{1}(\cdot)$ is a norm and we obtain the ideal of nuclear operators. When $E=F$ is a Hilbert space and $r=1$ the definition above agrees with the concept of trace class operators. For the case of Hilbert spaces $H$, the set of $r$-nuclear operators agrees with the Schatten-von Neumann class of order $r$ (see [34]).

The purpose of this paper is thus the study of the $r$-nuclearity of global pseudodifferential operators defined on Besov spaces in compact Lie groups [40], these operators can be defined as follows: let us assume that $G$ is a compact Lie group and denote by $\widehat{G}$ its unitary dual, i.e. the set of equivalence classes of all strongly continuous irreducible unitary representations of $G$. If $T$ is a linear operator from $C^{\infty}(G)$ into $C^{\infty}(G)$ and $\xi: G \rightarrow U\left(H_{\xi}\right)$ denotes an irreducible unitary representation, we can associate to $T$ a matrix-valued symbol $a(x, \xi) \in \mathbb{C}^{d_{\xi} \times d_{\xi}}$ (see (2.2)) satisfying

$$
T f(x)=\sum_{[\xi] \in \widehat{G}} d_{\xi} \operatorname{Tr}[\xi(x) a(x, \xi)(\mathscr{F} f)(\xi)],
$$

where in the summations is understood that from each class $[\xi]$ we pick just one representative $\xi \in[\xi], d_{\xi}=\operatorname{dim}\left(H_{\xi}\right)$ and $(\mathscr{F} f)(\xi)$ is the Fourier transform at $\xi$ :

$$
(\mathscr{F} f)(\xi):=\widehat{f}(\xi)=\int_{G} f(x) \xi(x)^{*} d x \in \mathbb{C}^{d_{\xi} \times d_{\xi}} .
$$

We are interested in the problem of the (nuclear) trace and trace formulae for $r$-nuclear pseudo-differential operators acting on Besov spaces defined in compact Lie groups as in [40]. There are several possibilities, concerning the conditions to impose on a symbol $a(x, \xi)$, in the attempt to establish the $r$-nuclearity of the corresponding operator $T_{a}$ on Lebesgue spaces defined in compact Lie groups. This problem was considered by J. Delgado and M. W. Wong (c.f. [9]) in the commutative case of the torus $\mathbb{T}^{n}$. To the best of our knowledge, this is the first work on the nuclearity and $\frac{2}{3}$-nuclearity of pseudo-differential operators on the torus.

It is a well known fact that the approximation property on a Banach space is 
required to define the nuclear trace [34]. A Banach space $E$ is said to have the approximation property if for every compact subset $K$ of $E$ and every $\varepsilon>0$ there exists a finite bounded operator $B$ on $E$ such that

$$
\|x-B x\|<\varepsilon, \text { for all } x \in K .
$$

On such spaces, if $T: E \rightarrow E$ is nuclear, the (nuclear) trace is defined by

$$
\operatorname{Tr}(T)=\sum_{n} e_{n}^{\prime}\left(y_{n}\right)
$$

where $T=\sum_{n} e_{n}^{\prime} \otimes y_{n}$ is a representation of $T$. If in the definition above $\|B\| \leq 1$, one says that $E$ has the metric approximation property. It is well known that every $L^{p}(\mu)$ space with $1 \leq p<\infty$ satisfies the approximation property. However, there exist Banach spaces which does not satisfy the approximation property. A counterexample to the statement that every Banach space $E$ has the approximation property was given early by P. Enflo in [20]. Enflo shows that there exists a separable reflexive Banach space with a sequence $M_{n}$ of finite dimensional subspaces with $\operatorname{dim}\left(M_{n}\right) \rightarrow \infty$, and a constant $c$ such that for every operator $T$ of finite rank, $\|T-I\| \geq 1-c\|T\| / \log \left(\operatorname{dim} M_{n}\right)$. We refer the reader to [4] for a work on subspaces of $l^{2}(X)$ without the approximation property. A remarkable result due to A. Grothendieck shows that for every $\frac{2}{3}$-nuclear operator $T$ acting on a Banach space $E$, the (nuclear) trace $\operatorname{Tr}(T)$ is well defined, the system of its eigenvalues is absolutely summable and the (nuclear) trace is equal to the sum of the eigenvalues (see [22], chapter II).

The $r$-nuclearity of operators give rise to results on the distribution of their eigenvalues (see [24]). This fact and the notion of spectral trace has been crucial in the study of spectral properties of nuclear operators arising in many different contexts and applications, such as the heat kernel on compact manifolds, the Fox-Li operator in laser engineering and transfer operators in thermodynamics (see [3, 7, 32].)

In this paper, which is based on the recent formulation of Besov spaces $B_{p, q}^{w}(G)$ on compact Lie groups given in [38], we prove that these spaces have the metric approximation property for $w \in \mathbb{R}, 1 \leq p<\infty$ and $1 \leq q \leq \infty$. Consequently, we derive a trace formula for $r$-nuclear operators in these spaces and, by using the compact Lie group structure of the $n$-dimensional torus $\mathbb{T}^{n}$, we prove some sufficient conditions for the $r$-nuclearity of periodic pseudo-differential operators on Besov spaces. The results are applied to study the corresponding trace formula of Grothendieck-Lidskii, which shows that the nuclear trace of these operators coincides with the spectral trace defined as the sum of eigenvalues. Similar results in the literature, on the $r$-nuclearity in $L^{p}$-spaces for pseudo-differential operators defined on compact Lie groups or on compact manifolds, can be found in the recent works of J. Delgado and M. Ruzhansky [9, 11, 13, 14] and references therein. The reference [19] consider the $r$-nuclearity of operators on manifolds with boundary. Mapping properties of pseudo-differential operators in Besov spaces defined on compact Lie groups can be found in [8]. 
There exist several recent works about the approximation property. In function spaces on euclidean domains as Lebesgue spaces with variable exponent, the space of functions of bounded variation, Sobolev spaces $W^{1,1}$, modulation spaces, Wiener-Amalgam spaces, and holomorphic functions on the disk, we refer the reader to $[2,6,16,17,18,27,37]$. Recent works on the approximation property for abstract Banach spaces can be found in [1, 25, 26, 28, 29, 30]. For a historical perspective on the approximation property we refer the reader to A. Pietsch [35].

We now describe the contents of the paper in more detail. In Section 2, Theorem 2.2, we present the metric approximation property for Besov spaces on compact Lie groups and some results are proved with respect to the $r$-nuclearity of Fourier multipliers. In Section 3, Theorem 3.3 and results therein provide sufficient conditions for the $r$-nuclearity of pseudo-differential operators acting on Besov spaces on the torus. Finally, in sections 4 and 5 , we establish trace formulae for $r$-nuclear periodic pseudo-differential operators on Besov spaces and specific periodic operators as negative powers of the Bessel potential and the heat kernel on the torus.

\section{The Metric APproximation Property FOR Besov SPACES AND NUCLEARITY OF FOURIER MULTIPLIERS IN COMPACT LIE GROUPS}

In this section we prove the metric approximation property for Besov spaces and we use this notion with the goal of investigate the nuclear trace of operators on Besov spaces. For the analysis on compact Lie groups, we refer the reader to $[40,41]$. See also [39] for a concise review of the theory on compact Lie groups. For the proof of the approximation property we use the following lemma (see e.g. [34]):

Lemma 2.1. A Banach space E satisfies the metric approximation property if, given $f_{1}, f_{2}, \cdots, f_{m} \in E$ and $\varepsilon>0$ there exists an operator of finite rank $B$ on $E$ such that $\|B\| \leq 1$ and

$$
\left\|f_{i}-B f_{i}\right\|<\varepsilon, \quad 1 \leq i \leq m
$$

Let us consider a compact Lie group $G$ with unitary dual $\widehat{G}$ that is, the set of equivalence classes of all strongly continuous irreducible unitary representations of $G$. We will equip $G$ with the Haar measure $\mu_{G}$ and, for simplicity, we will write $\int_{G} f(x) d x$ for $\int_{G} f d \mu_{G}, L^{p}(G)$ for $L^{p}\left(G, \mu_{G}\right)$, etc. The following equalities follow from the Fourier transform on $G$

$$
\widehat{f}(\xi)=\int_{G} f(x) \xi(x)^{*} d x, \quad f(x)=\sum_{[\xi] \in \widehat{G}} d_{\xi} \operatorname{Tr}(\xi(x) \widehat{f}(\xi)),
$$

and the Peter-Weyl Theorem on $G$ implies the Plancherel identity on $L^{2}(G)$,

$$
\|f\|_{L^{2}(G)}=\left(\sum_{[\xi] \in \widehat{G}} d_{\xi} \operatorname{Tr}\left(\widehat{f}(\xi) \widehat{f}(\xi)^{*}\right)\right)^{\frac{1}{2}}=\|\widehat{f}\|_{L^{2}(\widehat{G})^{\circ}}
$$


Notice that, since $\|A\|_{H S}=\operatorname{Tr}\left(A A^{*}\right)$, the term within the sum is the HilbertSchmidt norm of the matrix $A$. Any linear operator $T_{a}$ on $G$ mapping $C^{\infty}(G)$ into $\mathcal{D}^{\prime}(G)$ gives rise to a matrix-valued global (or full) symbol $a(x, \xi) \in \mathbb{C}^{d_{\xi} \times d_{\xi}}$ given by

$$
a(x, \xi)=\xi(x)^{*}(A \xi)(x),
$$

which can be understood from the distributional viewpoint. Then it can be shown that the operator $A=T_{a}$ can be expressed in terms of such a symbol as

$$
T_{a} f(x)=\sum_{[\xi] \in \widehat{G}} d_{\xi} \operatorname{Tr}[\xi(x) a(x, \xi) \widehat{f}(\xi)] .
$$

We introduce Sobolev and Besov spaces on compact Lie groups using the Fourier transform on the group $G$ as follows. There exists a non-negative real number $\lambda_{[\xi]}$ depending only on the equivalence class $[\xi] \in \hat{G}$, but not on the representation $\xi$, such that $-\mathcal{L}_{G} \xi(x)=\lambda_{[\xi]} \xi(x)$, where $\mathcal{L}_{G}$ is the Laplacian on the group $G$ (in this case, defined as the Casimir element on $G$ ). If we denote by $\langle\xi\rangle$ the function $\langle\xi\rangle=\left(1+\lambda_{[\xi]}\right)^{\frac{1}{2}}$, for every $s \in \mathbb{R}$ the Sobolev space $H^{s}(G)$ on the Lie group $G$ is defined by the condition: $f \in H^{s}(G)$ if only if $\langle\xi\rangle^{s} \widehat{f} \in L^{2}(\widehat{G})$. The Sobolev space $H^{s}(G)$ is a Hilbert space endowed with the inner product $\langle f, g\rangle_{s}=$ $\left\langle\left(I-\mathcal{L}_{G}\right)^{\frac{s}{2}} f,\left(I-\mathcal{L}_{G}\right)^{\frac{s}{2}} g\right\rangle_{L^{2}(G)}$, where, for every $s \in \mathbb{R},\left(I-\mathcal{L}_{G}\right)^{\frac{s}{2}}: H^{r} \rightarrow H^{r-s}$ is the bounded pseudo-differential operator with symbol $\langle\xi\rangle^{s} I_{\xi}$. Now, if $w \in \mathbb{R}$, $0<q \leq \infty$ and $0<p \leq \infty$, the Besov space $B_{p, q}^{w}(G)$ is the set of measurable functions on $G$ satisfying

$$
\|f\|_{B_{p, q}^{w}}:=\left(\sum_{m=0}^{\infty} 2^{m w q}\left\|\sum_{2^{m} \leq\langle\xi\rangle<2^{m+1}} d_{\xi} \operatorname{Tr}[\xi(x) \widehat{f}(\xi)]\right\|_{L^{p}(G)}^{q}\right)^{\frac{1}{q}}<\infty .
$$

If $q=\infty, B_{p, \infty}^{w}(G)$ consists of those functions $f$ satisfying

$$
\|f\|_{B_{p, \infty}^{w}}:=\sup _{m \in \mathbb{N}} 2^{m w}\left\|\sum_{2^{m} \leq\langle\xi\rangle<2^{m+1}} d_{\xi} \operatorname{Tr}[\xi(x) \widehat{f}(\xi)]\right\|_{L^{p}(G)}<\infty .
$$

A recent work on Besov spaces defined on homogeneous compact manifolds can be found in [38]. In the following theorem we present the metric approximation property for Besov spaces defined on arbitrary compact Lie groups.

Theorem 2.2. Let $G$ be a compact lie group. If $1 \leq p<\infty, 1 \leq q \leq \infty$ and $w \in \mathbb{R}$, then the Besov space $B_{p, q}^{w}(G)$ satisfies the metric approximation property.

Proof. First, we will prove the metric approximation property for $B_{p, q}^{w}(G)$. If $1 \leq p, q<\infty, B_{p, q}^{w}(G)$ is a Banach space. Let us consider $f_{1}, f_{2}, \cdots, f_{m} \in B_{p, q}^{w}(G)$. Then, by definition of Besov norm

$$
\left\|f_{i}\right\|_{B_{p, q}^{w}}^{q}=\sum_{s=0}^{\infty} 2^{s w q}\left\|\sum_{2^{s} \leq\langle\xi\rangle<2^{s+1}} d_{\xi} \operatorname{Tr}\left[\xi(x) \widehat{f}_{i}(\xi)\right]\right\|_{L^{p}}^{q}<\infty
$$


Let us consider the operator $T_{N}$ on $B_{p, q}^{w}(G)$ defined by

$$
\begin{aligned}
S_{N} f(x) & :=\sum_{\langle\xi\rangle \leq N} d_{\xi} \operatorname{Tr}(\xi(x) \widehat{f}(\xi)) \\
& =\sum_{\langle\xi\rangle \leq N} \sum_{i, j=1}^{d_{\xi}} d_{\xi} \xi_{i j}(x) \widehat{f}(\xi)_{j i},
\end{aligned}
$$

where the summation is understood that from each class $[\xi]$ we pick just one representative $\xi \in[\xi]$. Clearly, for every $N$ the operator $S_{N}$ has finite rank. Moreover,

$$
\operatorname{Rank}\left(S_{N}\right)=\operatorname{span}\left\{\xi_{i, j}(x): 1 \leq i, j \leq d_{\xi},\langle\xi\rangle \leq N\right\}
$$

Now, let $T_{N}=\left\|S_{N}\right\|^{-1} S_{N}$. Clearly, $\left\|T_{N}\right\|=1$ for every $N$. On the other hand, $T_{N}$ is a Fourier multiplier with matrix valued symbol

$$
\sigma_{N}(\xi)=\left\|S_{N}\right\|^{-1} \chi_{\{\xi:\langle\xi\rangle \leq N\}} I_{d_{\xi}}
$$

where $I_{d_{\xi}}$ is the matrix identity on $\mathbb{C}^{d_{\xi} \times d_{\xi}}$. We observe that

$$
\left\|f_{i}-T_{N} f_{i}\right\|_{B_{p, q}^{w}}^{q}=\sum_{s=0}^{\infty} 2^{s w q} \| \sum_{2^{s} \leq\langle\xi\rangle<2^{s+1}} d_{\xi} \operatorname{Tr}\left[\xi(x)\left(\widehat{f}_{i}(\xi)-\sigma_{N}(\xi) \widehat{f}_{i}(\xi)\right] \|_{L^{p}}^{q}\right.
$$

Using the fact that $S_{N}$ converges in the operator norm to the identity operator on $B_{p, q}^{w}$ we have $\lim _{N}\left\|S_{N}\right\|=1$. Observing that $\lim _{N \rightarrow \infty} \chi_{\{\xi:\langle\xi\rangle \leq N\}}(\eta)=1,[\eta] \in \widehat{G}$, and by using the convergence dominated theorem we obtain

$$
\lim _{N \rightarrow \infty}\left\|f_{i}-T_{N} f_{i}\right\|_{B_{p, q}^{w}}^{q}=0
$$

So, if $\varepsilon>0$ is given, for every $i$ there exists $N_{i}$ such that, if $N \geq N_{i}$ then

$$
\left\|f_{i}-T_{N} f_{i}\right\|_{B_{p, q}^{w}}<\varepsilon
$$

Thus, if

$$
M=\max \left\{N_{i}: 1 \leq i \leq m\right\}
$$

we have $\left\|T_{M}\right\|=1$ and $\left\|f_{i}-T_{M} f_{i}\right\|_{B_{p, q}^{w}}<\varepsilon$ for $1 \leq i \leq m$. So, by applying Lemma $2.1, B_{p, q}^{w}(G)$ has the metric approximation property for $w \in \mathbb{R}, 1 \leq p<\infty$ and $1 \leq q \leq \infty$. The result for $q=\infty$ has an analogous proof.

Now, we investigate the nuclear trace of Fourier multipliers on compact Lie groups. The following theorem will be an useful tool in order to establish the $r$ nuclearity of operators in Besov spaces. (An analogous result on Sobolev spaces has been proved in [12], Theorem 3.11).

Theorem 2.3. Let $G$ be a compact Lie group, $0<r \leq 1,1 \leq p<\infty$ and $1 \leq q \leq \infty$. Let us consider $T_{a}: C^{\infty}(G) \rightarrow C^{\infty}(G)$ be a Fourier multiplier with matrix valued symbol $a(\xi)$. The following two announcement are equivalent.

- (1) $T_{a}$ extends to a r-nuclear operator from $B_{p, q}^{w_{0}}(G)$ into $B_{p, q}^{w_{0}}(G)$ for some $w_{0} \in \mathbb{R}$.

- (2) $T_{a}$ extends to a r-nuclear operator from $B_{p, q}^{w}(G)$ into $B_{p, q}^{w}(G)$ for all $w \in \mathbb{R}$.

In this case, the nuclear trace of $T_{a}$ is independent of the index $w \in \mathbb{R}$. 
Proof. It is clear that (2) implies (1). Now, we will prove that (1) implies (2). Let us assume that $w, w_{0} \in \mathbb{R}, w \neq w_{0}$ and $T_{a}: B_{p, q}^{w_{0}}(G) \rightarrow B_{p, q}^{w_{0}}(G)$ is $r$-nuclear. The operators $\left(I-\mathcal{L}_{G}\right)^{\frac{w_{0}-w}{2}}: B^{w_{0}}(G) \rightarrow B^{w}(G)$ and $\left(I-\mathcal{L}_{G}\right)^{\frac{w-w_{0}}{2}}: B_{p, q}^{w}(G) \rightarrow$ $B_{p, q}^{w_{0}}(G)$ are both an isomorphism of Besov spaces. By considering that the class of $r$-nuclear operators is an ideal on the set of bounded operators, the following factorization of $T_{a}$ :

$$
T_{a}=\left(I-\mathcal{L}_{G}\right)^{\frac{w_{0}-w}{2}} \circ T_{a} \circ\left(I-\mathcal{L}_{G}\right)^{\frac{w-w_{0}}{2}}: B_{p, q}^{w}(G) \rightarrow B_{p, q}^{w}(G)
$$

shows that $T_{a}$ from $B_{p, q}^{w}(G)$ into $B_{p, q}^{w}(G)$ is $r$-nuclear. In the factorization above we have used that $T_{a}$ is a Fourier multiplier. Now, we will prove that the spectral trace of $T_{a}$ is independent of $w$. In fact, let us consider a nuclear representation of $T_{a}: B_{p, q}^{w_{0}} \rightarrow B_{p, q}^{w_{0}}:$

$$
T_{a} f=\sum_{n} e_{n}^{\prime}(f) y_{n}
$$

where $\left(e_{n}^{\prime}\right)_{n} \subset\left(B_{p, q}^{w_{0}}\right)^{\prime}$ and $\left(y_{n}\right)_{n} \subset B_{p, q}^{w_{0}}$ are sequences satisfying

$$
\sum_{n}\left\|e_{n}\right\|_{\left(B_{p, q}^{w_{0}}\right)^{\prime}}^{r}\left\|y_{n}\right\|_{B_{p, q}^{w_{0}}}^{r}<\infty .
$$

By Theorem 2.2 the space $B_{p, q}^{w_{0}}(G)$ has the approximation property and the nuclear trace of $T_{a}$ is well defined. Since

$$
\begin{aligned}
T_{a} f & =\left(I-\mathcal{L}_{G}\right)^{\frac{w_{0}-w}{2}} \circ T_{a} \circ\left(I-\mathcal{L}_{G}\right)^{\frac{w-w_{0}}{2}} f \\
& =\sum_{n}\left[e_{n}^{\prime} \circ\left(I-\mathcal{L}_{G}\right)^{\frac{w-w_{0}}{2}}(f)\right]\left(I-\mathcal{L}_{G}\right)^{\frac{w_{0}-w}{2}}\left(y_{n}\right)
\end{aligned}
$$

and

$$
\begin{aligned}
\sum_{n} & \left\|e_{n}^{\prime} \circ\left(I-\mathcal{L}_{G}\right)^{\frac{w-w_{0}}{2}}\right\|_{\left(B_{p, q}^{w}\right)}^{r}\left\|\left(I-\mathcal{L}_{G}\right)^{\frac{w_{0}-w}{2}}\left(y_{n}\right)\right\|_{B_{p, q}^{w}}^{r} \\
& \leq \sum_{n}\left\|\left(I-\mathcal{L}_{G}\right)^{\frac{w-w_{0}}{2}}\right\|_{B\left(B_{p, q}^{w}, B_{p, q}^{w_{0}}\right)}\left\|\left(I-\mathcal{L}_{G}\right)^{\frac{w_{0}-w}{2}}\right\|_{B\left(B_{p, q}^{\left.w_{0}, B_{p, q}^{w}\right)}\right.}\left\|e_{n}\right\|_{\left(B_{p, q}^{w_{0}}\right)^{\prime}}^{r}\left\|y_{n}\right\|_{B_{p, q}^{w_{0}}}^{r} \\
& =\left\|\left(I-\mathcal{L}_{G}\right)^{\frac{w-w_{0}}{2}}\right\|_{B\left(B_{p, q}^{w}, B_{p, q}^{w}\right)}^{w_{0}}\left\|\left(I-\mathcal{L}_{G}\right)^{\frac{w_{0}-w}{2}}\right\|_{B\left(B_{p, q}^{\left.w_{0}, B_{p, q}^{w}\right)}\right.} \sum_{n}\left\|e_{n}\right\|_{\left(B_{p, q}^{\left.w_{0}\right)^{\prime}}\right.}^{r}\left\|y_{n}\right\|_{B_{p, q}^{w_{0}}}^{r} \\
& <\infty
\end{aligned}
$$

we obtain that the nuclear trace of $T_{a}: B_{p, q}^{w} \rightarrow B_{p, q}^{w}$ is given by

$$
\operatorname{Tr}\left(T_{a}\right)=\sum_{n} e_{n}^{\prime} \circ\left(I-\mathcal{L}_{G}\right)^{\frac{w-w_{0}}{2}} \circ\left(I-\mathcal{L}_{G}\right)^{\frac{w_{0}-w}{2}}\left(y_{n}\right)=\sum_{n} e_{n}^{\prime}\left(y_{n}\right),
$$

which is the nuclear trace of $T_{a}: B_{p, q}^{w_{0}} \rightarrow B_{p, q}^{w_{0}}$. Thus, we end the proof.

As an application of Theorem 2.3, we obtain the following Theorem on the $r$-nuclearity and the $r$-nuclear trace of Fourier multipliers on $B_{p, q}^{w}(G)$. In order to present our theorem, we recall the following notation for the $l^{r}$-seminorm of matrices $A \in \mathbb{C}^{d \times d}$,

$$
\|A\|_{l^{r}}^{r}=\sum_{1 \leq i, j \leq d}\left|a_{i j}\right|^{r}, 0<r \leq 1
$$


and we define the following function,

$$
\varepsilon(t)=\frac{1}{2}, 1<t \leq 2 \text {, and } \varepsilon(t)=\frac{1}{t}, t \geq 2,
$$

which arises of natural way in $L^{p}$-estimates of the entries $\xi_{i j}$ of representations $[\xi] \in \widehat{G}$. In fact, for $1 \leq p \leq \infty,\left\|\xi_{i j}\right\|_{L^{p}(G)} \leq d_{\xi}^{-\varepsilon(p)}$. (See, Lemma 2.5 of [11]).

Theorem 2.4. Let $G$ be a compact Lie group, $n=\operatorname{dim}(G), 0<r \leq 1$ and $T_{a}: C^{\infty}(G) \rightarrow C^{\infty}(G)$ be a operator with matrix valued symbol a $(\xi)$. Under almost one of the following conditions

(1). $1<p<q<\infty$ and

$$
\sum_{[\xi] \in \hat{G}}\langle\xi\rangle^{n\left(\frac{1}{p}-\frac{1}{q}\right) r}\|a(\xi)\|_{l^{r}}^{r} d_{\xi}^{1+r\left(1-\varepsilon(p)-\varepsilon\left(q^{\prime}\right)\right)}<\infty .
$$

(2). $1 \leq p<\infty, q=1$ and

$$
\sum_{[\xi] \in \hat{G}}\langle\xi\rangle^{\frac{n r}{p}}\|a(\xi)\|_{l^{r}}^{r} d_{\xi}^{1+r(1-\varepsilon(p))}<\infty .
$$

(3). $1<p=q \leq 2$ and

$$
\sum_{[\xi] \in \hat{G}}\|a(\xi)\|_{l^{r}}^{r} d_{\xi}^{1+r\left(\frac{1}{p}-\frac{1}{2}\right)}<\infty
$$

(4). $2=q \leq p<\infty$ and

$$
\sum_{[\xi] \in \hat{G}}\|a(\xi)\|_{l^{r}}^{r} d_{\xi}^{1+r\left(\frac{1}{2}-\frac{1}{p}\right)}<\infty .
$$

The operator $T_{a}: B_{p, q}^{w} \rightarrow B_{p, q}^{w}$ extends to a $r$-nuclear operator for all $w \in \mathbb{R}$. Moreover, the nuclear trace of $T_{a}$ is given by

$$
\operatorname{Tr}\left(T_{a}\right)=\sum_{[\xi] \in \widehat{G}} d_{\xi} \operatorname{Tr}[a(\xi)]
$$

Proof. Let $T_{a}$ be the Fourier multiplier given by

$$
T_{a} f(x)=\sum_{[\xi] \in \widehat{G}} d_{\xi} \operatorname{Tr}[\xi(x) a(\xi)(\mathscr{F} f)(\xi)] .
$$

We observe that

$$
\operatorname{Tr}[\xi(x) a(\xi)(\mathscr{F} f)(\xi)]=\sum_{i, j . k=1}^{d_{\xi}} \xi(x)_{i, j} a(x, \xi)_{j, k}(\mathscr{F} f)(\xi)_{k, i} .
$$

Then, we can write

$$
T_{a} f(x)=\sum_{[\xi], i, j, k} H_{\xi, i, j, k}(x) G_{\xi, i, j, k}(f)
$$

where

$$
H_{\xi, i, j, k}(x)=d_{\xi} \xi(x)_{i j} a(\xi)_{i, k}, \quad G_{\xi, i, k}(f)=\widehat{f}(\xi)_{k i}, \quad 1 \leq i, j, k \leq d_{\xi} .
$$


Let us assume that $1<p, q<\infty$. We will prove that $T_{a}: B_{p, q}^{w}(G) \rightarrow B_{p, q}^{w}(G)$ is a $r$-nuclear in every case above by showing that

$$
\sum_{[\xi] \in \hat{G}} \sum_{i, j, k=1}^{d_{\xi}}\left\|H_{\xi, i, j, k}\right\|_{B_{p, q}^{w}}^{r}\left\|G_{\xi, i, j, k}\right\|_{\left(B_{p, q}^{w}\right)^{\prime}}^{r}<\infty .
$$

Later, considering the Theorem 2.3 we deduce the nuclearity of $T_{a}$ on $B_{p, q}^{w}$ for every $w \in \mathbb{R}$. First we estimate the Besov norm $\left\|H_{\xi, i, j, k}\right\|_{B_{p, q}^{w}}$ as follows:

$$
\begin{aligned}
& \left\|H_{\xi, i, j, k}\right\|_{B_{p, 1}^{w}}^{q}=\sum_{s=0}^{\infty} 2^{s w q}\left\|\sum_{2^{s} \leq\langle\eta\rangle<2^{s+1}} d_{\eta} \operatorname{Tr}\left[\eta(x) \widehat{H}_{\xi, i, j, k}(\eta)\right]\right\|_{L^{p}}^{q} \\
& =\sum_{s=0}^{\infty} 2^{s w q}\left\|\sum_{2^{s} \leq\langle\eta\rangle<2^{s+1}} d_{\eta} \operatorname{Tr}\left[\eta(x) \int_{G} \eta(y)^{*} d_{\xi} \xi(y)_{i j} a(\xi)_{j k} d y\right]\right\|_{L^{p}}^{q} \\
& =\sum_{s=0}^{\infty} 2^{s w q}\left\|\sum_{2^{s} \leq\langle\eta\rangle<2^{s+1}} \sum_{u, v=1}^{d_{\eta}} d_{\eta}\left[\eta(x)_{u v} \int_{G} \eta(y)_{v u}^{*} d_{\xi} \xi(y)_{i j} a(\xi)_{j k} d y\right]\right\|_{L^{p}}^{q} \\
& =\sum_{s=0}^{\infty} 2^{s w q}\left\|\sum_{2^{s} \leq\langle\eta\rangle<2^{s+1}} \sum_{u, v=1}^{d_{\eta}} d_{\eta}\left[\eta(x)_{u v} \int_{G} \overline{\eta(y)_{u v}} d_{\xi} \xi(y)_{i j} a(\xi)_{j k} d y\right]\right\|_{L^{p}}^{q} \\
& =\sum_{s=0}^{\infty} 2^{s w q}\left\|\sum_{2^{s} \leq\langle\eta\rangle\left\langle 2^{s+1}\right.} \sum_{u, v=1}^{d_{\eta}} d_{\eta}\left[\eta(x)_{u v} d_{\xi} a(\xi)_{j k}\left\langle\xi_{i j}, \eta_{u v}\right\rangle_{L^{2}(G)}\right]\right\|_{L^{p}}^{q} \\
& =\sum_{s=0}^{\infty} 2^{s w q}\left\|\sum_{2^{s} \leq\langle\eta\rangle<2^{s+1}} \sum_{u, v=1}^{d_{\eta}} d_{\eta}\left[\eta(x)_{u v} d_{\xi} a(\xi)_{j k} d_{\xi}^{-1 / 2} d_{\eta}^{-1 / 2} \delta_{(\xi, i, j),(\eta, u, v)}\right]\right\|_{L^{p}}^{q} .
\end{aligned}
$$

Let us chose the most smallest $s_{\xi} \in \mathbb{N}$ such that $2^{s_{\xi}} \leq\langle\xi\rangle<2^{s_{\xi}+1}$, considering that $\delta_{(\xi, i, j),(\eta, u, v)}=1$ only if $\xi=\eta, u=i$ and $v=j$ and $\delta_{(\xi, i, j),(\eta, u, v)}=0$ in other case, we obtain

$$
\left\|H_{\xi, i, j, k}\right\|_{B_{p, q}^{w}}=2^{s_{\xi} w} d_{\xi}\left\|\xi_{i j}\right\|_{L^{p}}\left|a(\xi)_{j k}\right|
$$

Since the $L^{p}$ norm of $\xi_{i j}$ can be estimate by $d_{\xi}^{-\varepsilon(p)}$ for $1<p<\infty$ and by considering that $2^{s_{\xi}} \leq\langle\xi\rangle<2^{s_{\xi}+1}$ we get

$$
\left\|H_{\xi, i, j, k}\right\|_{B_{p, q}^{w}} \leq\langle\xi\rangle^{w}\left|a(\xi)_{j k}\right| d_{\xi}^{1-\varepsilon(p)} .
$$


If $1<p<q<\infty$ and $w=n\left(\frac{1}{p}-\frac{1}{q}\right)$, we have the embedding $B_{p, q}^{w} \hookrightarrow L^{q}$ and we get,

$$
\begin{aligned}
\left\|G_{\xi, i, j, k}\right\|_{\left(B_{p, q}^{w}\right)^{\prime}} & =\sup _{\|f\|_{B_{p, q}^{w}=1}^{w}}\left|\widehat{f}(\xi)_{k i}\right| \leq \sup _{\|f\|_{B_{p, q}^{w}}^{w}=1}\left|\int_{G} \xi(x)_{k i}^{*} f(x) d x\right| \\
& \leq \sup _{\|f\|_{B_{p, q}^{w}}^{w}=1}\left\|\xi_{k i}^{*}\right\|_{L^{q^{\prime}}}\|f\|_{L^{q}} \lesssim \sup _{\|f\|_{B_{p, q}^{w}=1}} d_{\xi}^{-\varepsilon\left(q^{\prime}\right)}\|f\|_{L^{q}} \\
& \leq \sup _{\|f\|_{B_{p}^{w},}^{w}=1} d_{\xi}^{-\varepsilon\left(q^{\prime}\right)}\|f\|_{B_{p, q}^{w}} \\
& =d_{\xi}^{-\varepsilon\left(q^{\prime}\right)} .
\end{aligned}
$$

Now we estimate (2.12) as follows.

$$
\begin{aligned}
\sum_{[\xi] \in \hat{G}} \sum_{i, j, k=1}^{d_{\xi}}\left\|H_{\xi, i, j, k}\right\|_{B_{p, q}^{w}}^{r}\left\|G_{\xi, i, j, k}\right\|_{\left(B_{p, q}^{w}\right)^{\prime}}^{r} & \lesssim \sum_{[\xi] \in \hat{G}} \sum_{i, j, k=1}^{d_{\xi}}\langle\xi\rangle^{w r}\left|a(\xi)_{j k}\right|^{r} d_{\xi}^{r\left(1-\varepsilon(p)-\varepsilon\left(q^{\prime}\right)\right)} \\
& \lesssim \sum_{[\xi] \in \hat{G}} \sum_{j, k=1}^{d_{\xi}}\langle\xi\rangle^{w r}\left|a(\xi)_{j k}\right|^{r} d_{\xi}^{1+r\left(1-\varepsilon(p)-\varepsilon\left(q^{\prime}\right)\right)} \\
& \leq \sum_{[\xi] \in \hat{G}}\langle\xi\rangle^{w r}\|a(\xi)\|_{l^{r}}^{r} d_{\xi}^{1+r\left(1-\varepsilon(p)-\varepsilon\left(q^{\prime}\right)\right)}<\infty
\end{aligned}
$$

If we consider $1 \leq p<\infty$ and $q=1$ we have the embedding $B_{p, 1}^{w} \hookrightarrow L^{\infty}$ for $w=\frac{n}{p}$, and taking into account that $\left\|\xi_{k i}\right\|_{L^{\infty}} \leq 1$ we deduce the estimates

$$
\left\|H_{\xi, i, j, k}\right\|_{B_{p, 1}^{w}} \leq\langle\xi\rangle^{w}\left|a(\xi)_{j k}\right| d_{\xi}^{1-\varepsilon(p)}, \quad\left\|G_{\xi, i, j, k}\right\|_{\left(B_{p, 1}^{w}\right)^{\prime}} \leq \sup _{\substack{\frac{n}{p} \\ B_{p, \infty}^{p}=1}}\left\|\xi_{k i}^{*}\right\|_{L^{\infty}}\|f\|_{L^{\infty}} \lesssim 1 .
$$

So, we have

$$
\sum_{[\xi] \in \hat{G}} \sum_{i, j, k=1}^{d_{\xi}}\left\|H_{\xi, i, j, k}\right\|_{B_{p, 1}^{w}}^{r}\left\|G_{\xi, i, j, k}\right\|_{\left(B_{p, 1}^{w}\right)^{\prime}}^{r} \lesssim \sum_{[\xi] \in \hat{G}}\langle\xi\rangle^{w r}\|a(\xi)\|_{l^{r}}^{r} d_{\xi}^{1+r(1-\varepsilon(p))}<\infty
$$

The case where $1<p \leq 2$ we have the embedding $B_{p, p}^{w} \hookrightarrow H^{w, p}$, for every $w \in \mathbb{R}$. In particular, with $w=0$ we have the estimates

$$
\begin{aligned}
\sum_{[\xi] \in \hat{G}} \sum_{i, j, k=1}^{d_{\xi}}\left\|H_{\xi, i, j, k}\right\|_{B_{p, p}^{0}}^{r}\left\|G_{\xi, i, j, k}\right\|_{\left(B_{p, p}^{0}\right)^{\prime}}^{r} & \lesssim \sum_{[\xi] \in \hat{G}}\|a(\xi)\|_{l^{r}}^{r} d_{\xi}^{1+r\left(1-\frac{1}{2}-\frac{1}{p^{\prime}}\right)} \\
& \lesssim \sum_{[\xi] \in \hat{G}}\|a(\xi)\|_{l^{r}}^{r} d_{\xi}^{1+r\left(\frac{1}{p}-\frac{1}{2}\right)}<\infty
\end{aligned}
$$


Now, for $q=2 \leq p<\infty$ we use the embedding $B_{p, 2}^{0} \hookrightarrow H^{0, p}$ in order to obtain

$$
\begin{aligned}
\sum_{[\xi] \in \hat{G}} \sum_{i, j, k=1}^{d_{\xi}}\left\|H_{\xi, i, j, k}\right\|_{B_{p, 2}^{0}}^{r}\left\|G_{\xi, i, j, k}\right\|_{\left(B_{p, 2}^{0}\right)^{\prime}}^{r} & \lesssim \sum_{[\xi] \in \hat{G}}\|a(\xi)\|_{l^{r}}^{r} d_{\xi}^{1+r\left(1-\frac{1}{p}-\frac{1}{2}\right)} \\
& \lesssim \sum_{[\xi] \in \hat{G}}\|a(\xi)\|_{l^{r}}^{r} d_{\xi}^{1+r\left(\frac{1}{2}-\frac{1}{p}\right)}<\infty
\end{aligned}
$$

So, in every specific case, we have proved that $T_{a}$ is nuclear on $B_{p, q}^{w}$ and therefore on every $B_{p, 1}^{w}$ with $w \in \mathbb{R}$. Now, we compute the nuclear trace of $T_{a}$. Since $B_{p, q}^{w}$ has the approximation property, we deduce that the nuclear trace of $T_{a}$ is well defined, this means that it can be computed from any nuclear decomposition. So we get

$$
\operatorname{Tr}\left(T_{a}\right)=\sum_{[\xi], i, j, k} G_{\xi, i, k}\left(H_{\xi, i, j, k}\right)=\sum_{[\xi], i, j, k} \mathscr{F}\left(H_{\xi, i, j, k}\right)(\xi)_{k, i} .
$$

By using the definition of Fourier transform, we obtain

$$
\mathscr{F}\left(H_{\xi, i, j, k}\right)(\xi)=\int_{G} d_{\xi} \xi(x)^{*} \xi(x)_{i, j} a(\xi)_{j, k} d x .
$$

Hence

$$
\mathscr{F}\left(H_{\xi, i, j, k}\right)(\xi)_{k i}=\int_{G} d_{\xi} \xi(x)_{k, i}^{*} \xi(x)_{i, j} a(\xi)_{j, k} d x .
$$

Using this fact, we deduce that

$$
\begin{aligned}
\operatorname{Tr}\left(T_{a}\right) & =\sum_{[\xi], i, j, k} d_{\xi} \int_{G} \xi(x)_{k, i}^{*} \xi(x)_{i, j} a(\xi)_{j, k} d x \\
& =\sum_{[\xi] \in \widehat{G}} d_{\xi} \int_{G} \sum_{i, j, k=1}^{d_{\xi}} \xi(x)_{k, i}^{*} \xi(x)_{i, j} a(\xi)_{j, k} d x \\
& =\sum_{[\xi] \in \widehat{G}} d_{\xi} \int_{G} \operatorname{Tr}\left[\xi(x) a(\xi) \xi\left(x^{*}\right)\right] \\
& =\sum_{[\xi] \in \widehat{G}} d_{\xi} \operatorname{Tr}[a(\xi)] .
\end{aligned}
$$

Thus, we end the proof.

Remark 2.5. Now, we discuss the theorem above in relation with results in $L^{2}$ spaces. We observe that the result obtained when $p=q=2$ in the condition (3) of Theorem 2.4, is most weak that Theorem 3.1 of [11] where the condition

$$
\sum_{[\xi] \in \hat{G}}\|a(\xi)\|_{S_{r}}^{r} d_{\xi}<\infty
$$

is imposed in terms of the $r$-Schatten seminorm $\|a(\xi)\|_{S_{r}}$ in order to obtain $r$ nuclearity. It was mentioned in 2.4 that such condition is also necessary for the $r$-nuclearity of $T_{a}$. There exists two cases where both results are equivalent. One 
is, the case where the operator $T_{a}: C^{\infty}(G) \rightarrow C^{\infty}(G)$ is formally self-adjoint. In fact, with such condition in mind, one can to assume that the corresponding symbol $a(\xi)$ is diagonal by choosing a suitable basis in the representations spaces. In a such case, $\|a(\xi)\|_{l^{r}}=\|(\xi)\|_{S_{r}}$. The other case arises when $G=\mathbb{T}^{n}$ is some $n$-dimensional torus, where $\widehat{G}=\mathbb{Z}^{n}$ and for every $\xi \in \mathbb{Z}^{n},\|a(\xi)\|_{l^{r}}=\|(\xi)\|_{S_{r}}=$ $|a(\xi)|$. It is important to mention that the trace formula obtained above coincides with ones for $r$-Fourier multiplier in $L^{p}$ spaces obtained in [11, 12].

We end this section with the following two examples on the nuclearity of suitable powers of the Bessel's potential and the heat operator.

Example 2.6. Let $G$ be a compact Lie group, $\mathcal{L}_{G}$ be the Laplace-Beltrami operator on $G$ and $n=\operatorname{dim}(G)$. We note that as consequence of Theorem 2.4, if $\alpha>n$ and $1<p \leq 2$, the operator $T_{a}=\left(1-\mathcal{L}_{G}\right)^{-\frac{\alpha}{2}}$ is nuclear on $B_{p, 1}^{w}(G)$ for all $-\infty<w<\infty$. Indeed, this operator has symbol $a(\xi)$ satisfying the condition (3) of Theorem 2.4 and as consequence we get

$$
\sum_{[\xi] \in \hat{G}} d_{\xi}\|a(\xi)\|_{l^{1}}:=\sum_{[\xi] \in \hat{G}} d_{\xi}^{2}\langle\xi\rangle^{-\alpha}<\infty .
$$

In this case

$$
\operatorname{Tr}\left[\left(1-\mathcal{L}_{G}\right)^{-\frac{\alpha}{2}}\right]=\sum_{[\xi] \in \widehat{G}} d_{\xi}^{2}\langle\xi\rangle^{-\alpha} .
$$

Example 2.7. For $t>0$ the heat operator is defined by $e^{-t \mathcal{L}_{G}}$ and its symbol is given by $a_{t}(\xi)=e^{-t \lambda_{[\xi]}} I_{d_{\xi}}$. Clearly this symbol satisfies the hypotheses of Theorem 2.4 , and $T_{a}=e^{-t \mathcal{L}_{G}}$ is a nuclear operator on $B_{p, 1}^{w}(G)$ for all $-\infty<w<\infty$. For the heat operator, the nuclear trace is

$$
\operatorname{Tr}\left[e^{-t \mathcal{L}_{G}}\right]=\sum_{[\xi] \in \widehat{G}} d_{\xi}^{2} e^{-t \lambda_{[\xi]}}
$$

\section{3. $r$-NUCLEAR PSEUDO-DIFFERENTIAL OPERATOR ON PERIODIC BESOV SPACES}

In this section we present our results on the $r$-nuclearity of pseudo-differential operators on periodic Besov spaces. We use the notation of periodic pseudodifferential operators as developed in [40]. Let us denote by $\mathcal{S}\left(\mathbb{Z}^{n}\right)$ the Schwartz space of functions $\phi: \mathbb{Z}^{n} \rightarrow \mathbb{C}$ such that

$$
\forall M \in \mathbb{R}, \exists C_{M}>0,|\phi(\xi)| \leq C_{M}\langle\xi\rangle^{M},
$$

where $\langle\xi\rangle=\left(1+|\xi|^{2}\right)^{\frac{1}{2}}$. The toroidal Fourier transform is defined, for any $f \in$ $C^{\infty}\left(\mathbb{T}^{n}\right)$, by $\widehat{f}(\xi)=\int e^{-i 2 \pi\langle x, \xi\rangle} f(x) d x$, where $\xi \in \mathbb{Z}^{n}$, and the inversion formula is given by $f(x)=\sum e^{i 2 \pi\langle x, \xi\rangle} \widehat{u}(\xi)$, for $x \in \mathbb{T}^{n}$. The periodic Hörmander class $S_{\rho, \delta}^{m}\left(\mathbb{T}^{n} \times \mathbb{R}^{n}\right), 0 \leq \rho, \delta \leq 1$, consists of those functions $a(x, \xi)$ which are smooth in $(x, \xi) \in \mathbb{T}^{n} \times \mathbb{R}^{n}$ and which satisfy toroidal symbols inequalities

$$
\left|\partial_{x}^{\beta} \partial_{\xi}^{\alpha} a(x, \xi)\right| \leq C_{\alpha, \beta}\langle\xi\rangle^{m-\rho|\alpha|+\delta|\beta|} .
$$


Symbols in $S_{\rho, \delta}^{m}\left(\mathbb{T}^{n} \times \mathbb{R}^{n}\right)$ are symbols in $S_{\rho, \delta}^{m}\left(\mathbb{R}^{n} \times \mathbb{R}^{n}\right)$ (see [40]) of order $m$ which are 1-periodic in $x$. If $a(x, \xi) \in S_{\rho, \delta}^{m}\left(\mathbb{T}^{n} \times \mathbb{R}^{n}\right)$, the corresponding pseudodifferential operator is defined by

$$
T_{a} u(x)=\int_{\mathbb{T}^{n}} \int_{\mathbb{R}^{n}} e^{i 2 \pi\langle x-y, \xi\rangle} a(x, \xi) u(y) d \xi d y .
$$

The set $S_{\rho, \delta, \nu, \mu}^{m}\left(\mathbb{T}^{n} \times \mathbb{Z}^{n}\right), 0 \leq \rho, \delta \leq 1, \nu, \mu \in \mathbb{N}$, consists of those functions $a(x, \xi)$ which are smooth in $x$ for all $\xi \in \mathbb{Z}^{n}$ and which satisfy

$$
\left|\Delta_{\xi}^{\alpha} \partial_{x}^{\beta} a(x, \xi)\right| \leq C_{\alpha, \beta}\langle\xi\rangle^{m-\rho|\alpha|+\delta|\beta|}, \quad|\alpha| \leq \nu,|\beta| \leq \mu .
$$

The operator $\Delta$ is the difference operator defined in [40]. The toroidal operator with symbol $a(x, \xi)$ is defined as

$$
a\left(x, D_{x}\right) u(x)=\sum_{\xi \in \mathbb{Z}^{n}} e^{i 2 \pi\langle x, \xi\rangle} a(x, \xi) \widehat{u}(\xi), u \in C^{\infty}\left(\mathbb{T}^{n}\right) .
$$

Besov spaces have been introduced in Section 2 for general compact Lie groups. Now we present this notion for the toroidal case; let $w \in \mathbb{R}, 0<q<\infty$ and $0<p \leq \infty$. If $f$ is a measurable function on $\mathbb{T}^{n}$, we say that $f \in B_{p, q}^{w}\left(\mathbb{T}^{n}\right)$ if $f$ satisfies

$$
\|f\|_{B_{p, q}^{w}}:=\left(\sum_{m=0}^{\infty} 2^{m w q}\left\|\sum_{2^{m} \leq|\xi|<2^{m+1}} e^{i 2 \pi x \cdot \xi} \widehat{f}(\xi)\right\|_{L^{p}\left(\mathbb{T}^{n}\right)}^{q}\right)^{\frac{1}{q}}<\infty .
$$

If $q=\infty, B_{p, \infty}^{w}\left(\mathbb{T}^{n}\right)$ consists of those functions $f$ satisfying

$$
\|f\|_{B_{p, \infty}^{w}}:=\sup _{m \in \mathbb{N}} 2^{m w}\left\|\sum_{2^{m} \leq|\xi|<2^{m+1}} e^{i 2 \pi x \xi} \widehat{f}(\xi)\right\|_{L^{p}\left(\mathbb{T}^{n}\right)}<\infty .
$$

In the case of $p=q=\infty$ and $0<w<1=n$ we obtain $B_{\infty, \infty}^{w}(\mathbb{T})=\Lambda^{w}(\mathbb{T})$, that is the Hölder space of order $\omega$; these are Banach spaces together with the norm

$$
\|f\|_{\Lambda^{w}}=\sup _{x, h \in \mathbb{T}}|f(x+h)-f(x)||h|^{-w}+\sup _{x \in \mathbb{T}}|f(x)| .
$$

For $1 \leq p \leq \infty$ and $1 \leq q \leq \infty, B_{p, q}^{w}\left(\mathbb{T}^{n}\right)$ are Banach spaces. Moreover, if $w \in \mathbb{R}$ we have the identity of Hilbert spaces $H_{2,2}^{w}\left(\mathbb{T}^{n}\right)=B_{2,2}^{r}\left(\mathbb{T}^{n}\right)$ of Besov spaces with Sobolev spaces. When studying the orders of periodic pseudo-differential operators, estimates for the Fourier coefficients of symbols are useful, and therefore we present an auxiliary result on this subject.

Lemma 3.1. Let $0 \leq \rho, \delta \leq 1$. Assume that $a \in S_{\rho, \delta, u, 2 k}^{m}\left(\mathbb{T}^{n} \times \mathbb{Z}^{n}\right)$. Let $\widehat{a}(\eta, \cdot)$ be the Fourier transform of the symbol with respect to $x$, i.e., the Fourier transform of the smooth function $x \mapsto a(x, \cdot)$. Then, for every $k \in \mathbb{N}$ we have

$$
|\widehat{a}(\eta, \xi)| \leq C\langle\eta\rangle^{-2 k}\langle\xi\rangle^{m+\delta|2 k|} .
$$

Proof. The proof can be found in [40], Lemma 4.2.1. 
With notation above, we present our results on the $r$-nuclearity of periodic pseudo-differential operators. We reserve the notation $A \lesssim B$ if there exists $c>0$ independent of $A$ and $B$ such that $A \leq c \cdot B$. The conjugate exponent $p^{\prime}$ of $p$, $1 \leq p \leq \infty$ is defined by $1 / p+1 / p^{\prime}=1$. Our starting point is the following result (Theorem 6.2 of [38]).

Lemma 3.2. Let $1<p_{1} \leq 2, \alpha>0, n \in \mathbb{N}$ and $\beta=\left(\alpha+\frac{1}{p_{1}^{\prime}}\right)^{-1}$. If $w_{1}=\alpha$ then the Fourier transform is a bounded operator from $B_{p_{1}, \beta}^{\alpha n}\left(\mathbb{T}^{n}\right)$ into $L^{\beta}\left(\mathbb{Z}^{n}\right)$.

By using the lemma above, we obtain the following result on the $r$-nuclearity of pseudo-differential operators on periodic Besov spaces.

Theorem 3.3. Let $0<r \leq 1,0<\alpha \leq \frac{1}{2}, 0 \leq \rho, \delta \leq 1$, and $k>\frac{n}{2}, k \in \mathbb{N}$. Let us consider $a \in S_{\rho, \delta, 0,2 k}^{m}\left(\mathbb{T}^{n} \times \mathbb{Z}^{n}\right)$. Under the following conditions,

- $w_{1}=\alpha \cdot n, 1<p_{1} \leq 2, q_{1}=\left(\alpha+\frac{1}{p_{1}^{\prime}}\right)^{-1}$.

- $0 \leq w_{2}<2 k-n, m<-\frac{n}{r}-w_{2}-\delta(2 k), 1 \leq p_{2} \leq \infty, 1 \leq q_{2} \leq \infty$,

the pseudo-differential operator $T_{a}: B_{p_{1}, q_{1}}^{w_{1}}\left(\mathbb{T}^{n}\right) \rightarrow B_{p_{2}, q_{2}}^{w_{2}}\left(\mathbb{T}^{n}\right)$ is r-nuclear.

Proof. We begin by writing

$$
T_{a} f(x)=\sum_{\xi \in \mathbb{Z}^{n}} e^{i 2 \pi x \cdot \xi} a(x, \xi) \widehat{f}(\xi)=\sum_{\xi \in \mathbb{Z}^{n}} G_{\xi}(f) H_{\xi}(x),
$$

where $H_{\xi}(x)=e^{i 2 \pi x \xi} a(x, \xi)$ and $G_{\xi}(f)=\widehat{f}(\xi)$. By Lemma 3.2, for every $\xi \in \mathbb{Z}^{n}$ we have

$$
\begin{aligned}
\left\|G_{\xi}\right\|_{\left(B_{p_{1}, q_{1}}^{w_{1}}\right)^{\prime}} & =\sup \left\{\left|G_{\xi}(f)\right|:\|f\|_{B_{p_{1}, q_{1}}^{w_{1}}}=1\right\} \\
& =\sup \left\{|\widehat{f}(\xi)|:\|f\|_{B_{p_{1}, q_{1}}^{w_{1}}}=1\right\} \\
& \leq \sup \left\{\|\widehat{f}\|_{L^{\beta}\left(\mathbb{Z}^{n}\right)}:\|f\|_{B_{p_{1}, q_{1}}^{w_{1}}}=1\right\} \\
& \lesssim 1
\end{aligned}
$$

Now, if $1 \leq q_{2}<\infty$ we observe that

$$
\left\|H_{\xi}\right\|_{B_{p_{2}, q_{2}}^{w_{2}}}^{q_{2}}=\sum_{s=0}^{\infty} 2^{s w_{2} q_{2}}\left\|\sum_{2^{s} \leq|\eta|<2^{s+1}} e^{i 2 \pi \eta y} \widehat{H_{\xi}}(\eta)\right\|_{L^{p_{2}}}^{q_{2}},
$$

where

$$
\begin{aligned}
\widehat{H_{\xi}}(\eta) & =\int_{\mathbb{T}^{n}} e^{-i 2 \pi x \eta} e^{i 2 \pi x \xi} a(x, \xi) d x \\
& =\widehat{a}(\eta-\xi, \xi) .
\end{aligned}
$$


Hence, we have

$$
\begin{aligned}
\left\|H_{\xi}\right\|_{B_{p_{2}, q_{2}}^{w_{2}}} & \leq\left(\sum_{s=0}^{\infty} 2^{s w_{2} q_{2}}\left[\sum_{2^{s} \leq|\eta|<2^{s+1}}|\widehat{a}(\eta-\xi, \xi)|\right]^{q_{2}}\right)^{1 / q_{2}} \\
& \leq\left(\sum_{s=0}^{\infty}\left[\sum_{2^{s} \leq|\eta|<2^{s+1}}|\eta|^{w_{2}}|\widehat{a}(\eta-\xi, \xi)|\right]^{q_{2}}\right)^{1 / q_{2}} \\
& =\|F\|_{L^{q_{2}(\mathbb{N})}}
\end{aligned}
$$

where $F$ is the sequence on $\mathbb{N}$ given by

$$
F(s)=\sum_{2^{s} \leq|\eta|<2^{s+1}}|\eta|^{w_{2}}|\widehat{a}(\eta-\xi, \xi)| .
$$

Since $L^{1}(\mathbb{N}) \subset L^{q_{2}}(\mathbb{N})$ is a continuous inclusion, we have $\|F\|_{L^{q_{2}}} \lesssim\|F\|_{L^{1}}$. Hence,

$$
\begin{aligned}
\left\|H_{\xi}\right\|_{B_{p_{2}, q_{2}}^{w_{2}}} & \lesssim \sum_{s=0}^{\infty} \sum_{2^{s} \leq|\eta|<2^{s+1}}|\eta|^{w_{2}}|\widehat{a}(\eta-\xi, \xi)| \\
& \leq \sum_{\eta \in \mathbb{Z}^{n}}|\eta|^{w_{2}}|\widehat{a}(\eta-\xi, \xi)| .
\end{aligned}
$$

If $q_{2}=\infty$, by definition of Besov norm we have

$$
\begin{aligned}
\left\|H_{\xi}\right\|_{B_{p_{2}, q_{2}}^{w_{2}}} & =\sup _{s \in \mathbb{N}} 2^{s w_{2}}\left\|\sum_{2^{s} \leq|\eta|<2^{s+1}} e^{i 2 \pi \eta y} \widehat{H_{\xi}}(\eta)\right\|_{L^{p_{2}}} \\
& =\sup _{s \in \mathbb{N}} 2^{s w_{2}}\left\|\sum_{2^{s} \leq|\eta|<2^{s+1}} e^{i 2 \pi \eta y} \widehat{a}(\eta-\xi, \xi)\right\|_{L^{p_{2}}} \\
& \leq \sup _{s \in \mathbb{N}} 2^{s w_{2}} \sum_{2^{s} \leq|\eta|<2^{s+1}}|\widehat{a}(\eta-\xi, \xi)| \\
& \leq \sup _{s \in \mathbb{N}} \sum_{2^{s} \leq|\eta|<2^{s+1}}|\eta|^{w_{2}}|\widehat{a}(\eta-\xi, \xi)| \\
& \leq \sum_{\eta \in \mathbb{Z}^{n}}|\eta|^{w_{2}}|\widehat{a}(\eta-\xi, \xi)| .
\end{aligned}
$$

Now, by Lemma 3.1 we have $|\widehat{a}(\eta-\xi, \xi)| \leq C\langle\eta-\xi\rangle^{-2 k}\langle\xi\rangle^{m+\delta(2 k)}$. On the other hand, by Peetre's inequality (Proposition 3.3.31 of [40]) we can write

$$
|\eta|^{w_{2}} \lesssim\langle\eta\rangle^{w_{2}} \lesssim\langle\eta-\xi\rangle^{w_{2}}\langle\xi\rangle^{w_{2}}
$$

From this, for all $1 \leq q_{2} \leq \infty$ we obtain,

$$
\begin{aligned}
\left\|H_{\xi}\right\|_{B_{p_{2}, q_{2}}^{w_{2}}} & \leq \sum_{\eta \in \mathbb{Z}^{n}}\langle\eta-\xi\rangle^{w_{2}-2 k}\langle\xi\rangle^{w_{2}+m+\delta(2 k)} \\
& =\langle\xi\rangle^{w_{2}+m+\delta(2 k)} \sum_{\eta \in \mathbb{Z}^{n}}\langle\eta\rangle^{w_{2}-2 k}
\end{aligned}
$$


From the condition $0 \leq w_{2}<2 k-n$ we deduce the convergence of the series

$$
\sum_{\eta \in \mathbb{Z}^{n}}\langle\eta\rangle^{w_{2}-2 k}
$$

Hence, we have

$$
\sum_{\xi \in \mathbb{Z}^{n}}\left\|H_{\xi}\right\|_{B_{p_{2}, q_{2}}^{w_{2}}}^{r}\left\|G_{\xi}\right\|_{\left(B_{p_{1}, q_{1}}^{w_{1}}\right)^{\prime}}^{r} \lesssim \sum_{\xi \in \mathbb{Z}^{n}}\langle\xi\rangle^{r\left(w_{2}+m+\delta(2 k)\right)}<\infty .
$$

Since $0<\alpha \leq \frac{1}{2}$, we deduce that $q_{1} \geq 1$. Hence $B_{p_{1}, q_{1}}^{w_{1}}$ is a Banach space. So, $T_{a}: B_{p_{1}, q_{1}}^{w_{1}}\left(\mathbb{T}^{n}\right) \rightarrow B_{p_{2}, q_{2}}^{w_{2}}\left(\mathbb{T}^{n}\right)$ is a $r$-nuclear operator.

In the previous theorem the $r$-nuclearity has been established for $w_{2} \geq 0$. In the following theorem we provide an analysis of the problem for $w_{2}<0$.

Theorem 3.4. Let $0 \leq \delta, \rho \leq 1$, and $0<\alpha \leq \frac{1}{2}$. If $a \in S_{\rho, \delta, 0,2 k}^{m}$, under the following conditions

- $w_{1}=\alpha \cdot n, 1<p_{1} \leq 2, q_{1}=\left(\alpha+\frac{1}{p_{1}^{\prime}}\right)^{-1}$,

- $-\infty<w_{2}<-\frac{n}{2}, 1 \leq p_{2}, q_{2} \leq \infty, m \leq-\delta(2 k), k>n / 4$,

the operator $T_{a}: B_{p_{1}, q_{1}}^{w_{1}} \rightarrow B_{p_{2}, q_{2}}^{w_{2}}$ is nuclear. Moreover, if we assume

- $w_{1}=\alpha \cdot n, 1<p_{1} \leq 2, q_{1}=\left(\alpha+\frac{1}{p_{1}^{\prime}}\right)^{-1}$,

- $w_{2} \leq 0,1 \leq p_{2}, q_{2} \leq \infty, m<-\frac{n}{r}-\delta(2 k), k>n / 2$,

the operator $T_{a}$ is $r$-nuclear for all $0<r \leq 1$.

Proof. From the proof of Theorem 3.3, we have that $\left\|G_{\xi}\right\|_{B_{p_{1}, q_{1}}^{w_{1}}} \lesssim 1$ and

$$
\begin{aligned}
\left\|H_{\xi}\right\|_{B_{p_{2}, q_{2}}^{w_{2}}} & \lesssim \sum_{s=0}^{\infty} \sum_{2^{s} \leq|\eta|<2^{s+1}}|\eta|^{w_{2}}|\widehat{a}(\eta-\xi, \xi)| \\
& \leq \sum_{\eta \neq 0}|\eta|^{w_{2}}|\widehat{a}(\eta-\xi, \xi)| .
\end{aligned}
$$

If $-\infty<w_{2}<-n / 2$, we deduce that

$$
\begin{aligned}
\left\|H_{\xi}\right\|_{B_{p_{2}, q_{2}}^{w_{2}}} & \lesssim \sum_{\eta \in \mathbb{Z}^{n}}\langle\eta\rangle^{w_{2}}|\widehat{a}(\eta-\xi, \xi)| \\
& \lesssim \sum_{\eta \in \mathbb{Z}^{n}}\langle\eta\rangle^{w_{2}}\langle\eta-\xi\rangle^{-2 k}\langle\xi\rangle^{m+\delta(2 k)} \\
& =\langle\xi\rangle^{m+\delta(2 k)}\left[\langle\cdot\rangle^{w_{2}} *\langle\cdot\rangle^{-2 k}\right](\xi) .
\end{aligned}
$$

By the Young's inequality, $\langle\eta\rangle^{w_{2}} \in L^{2}(\mathbb{Z})$ and $\langle\eta\rangle^{-2 k} \in L^{2}(\mathbb{Z})$ implies that

$$
\langle\cdot\rangle^{w_{2}} *\langle\cdot\rangle^{-2 k} \in L^{1}(\mathbb{Z}) .
$$

With this in mind, using the fact that $\left\|G_{\xi}\right\|_{B_{p_{1}, q_{1}}^{w_{1}}} \lesssim 1$, and the condition $m \leq$ $-\delta(2 k)$ we obtain

$$
\sum_{\xi \in \mathbb{Z}^{n}}\left\|H_{\xi}\right\|_{B_{p_{2}, q_{2}}^{w_{2}}}\left\|G_{\xi}\right\|_{\left(B_{p_{1}, q_{1}}^{w_{1}}\right)^{\prime}} \lesssim \sum_{\xi \in \mathbb{Z}^{n}}\left[\langle\cdot\rangle^{w_{2}} *\langle\cdot\rangle^{-2 k}\right](\xi)<\infty
$$


This inequality implies the nuclearity of $T_{a}$. Now, we will treat the case $w_{2} \leq 0$, $k>n / 2$. In fact, we have

$$
\begin{aligned}
\left\|H_{\xi}\right\|_{B_{p_{2}, q_{2}}^{w_{2}}} & \lesssim \sum_{\eta \in \mathbb{Z}^{n}}\langle\eta\rangle^{w_{2}}|\widehat{a}(\eta-\xi, \xi)| \\
& \lesssim \sum_{\eta \in \mathbb{Z}^{n}}\langle\eta-\xi\rangle^{-2 k}\langle\xi\rangle^{m+\delta(2 k)} \\
& \lesssim\langle\xi\rangle^{m+\delta(2 k)}
\end{aligned}
$$

Thus,

$$
\sum_{\xi \in \mathbb{Z}^{n}}\left\|H_{\xi}\right\|_{B_{p_{2}, q_{2}}^{w_{2}}}^{r}\left\|G_{\xi}\right\|_{\left(B_{p_{1}, q_{1}}^{w_{1}}\right)^{\prime}}^{r} \lesssim \sum_{\xi \in \mathbb{Z}^{n}}\langle\xi\rangle^{r(m+\delta \cdot 2 k)}<\infty
$$

This proves the $r$-nuclearity of $T_{a}$ when $m<-\frac{n}{r}-\delta \cdot 2 k$.

In order to get $r$-nuclearity of operators from Hölder into Besov spaces, we recall the following lemma (see $[5,42,43]$.)

Lemma 3.5. Let $1 \leq p \leq 2$ and let $s_{p}=1 / p-1 / 2$. Then, the Fourier transform $f \mapsto \mathscr{F} f$ from $\Lambda^{s}(\mathbb{T})$ into $L^{p}(\mathbb{T})$ is a bounded operator for all $s, s_{p}<s<1$. In particular, if $p=1$ we obtain the Bernstein Theorem.

Now we study the $r$-nuclearity of periodic operators from Hölder spaces (resp. Lebesgue) into Besov spaces.

Theorem 3.6. Let $0<r \leq 1,0 \leq \rho, \delta \leq 1$, and $k>\frac{n}{2}, k \in \mathbb{N}$. Let us consider $a \in S_{\rho, \delta, 0,2 k}^{m}\left(\mathbb{T}^{n} \times \mathbb{Z}^{n}\right)$. Under the following conditions,

- $X^{(n)}=L^{p}\left(\mathbb{T}^{n}\right), 1 \leq p \leq 2$ or $X^{(1)}=B_{\infty, \infty}^{s}\left(\mathbb{T}^{1}\right), 0<s<1$.

- $0 \leq w_{2}<2 k-n, m<-\frac{n}{r}-w_{2}-\delta(2 k), 1 \leq p_{2} \leq \infty, 1 \leq q_{2} \leq \infty$,

the pseudo-differential operator $T_{a}: X^{(n)} \rightarrow B_{p_{2}, q_{2}}^{w_{2}}\left(\mathbb{T}^{n}\right)$ is r-nuclear.

Proof. If we assume that $X:=X\left(\mathbb{T}^{n}\right)$ is a Banach space of periodic functions with the property that $\|\widehat{f}\|_{L^{\infty}\left(\mathbb{Z}^{n}\right)} \leq C\|f\|_{X\left(\mathbb{T}^{n}\right)}$, then we obtain

$$
\left\|G_{\xi}\right\|_{X^{\prime}}:=\sup _{\|f\|_{X}=1}|\widehat{f}(\xi)| \leq C .
$$

Now, we note that in the following cases, $X$ has the mentioned property:

- $X=L^{1}\left(\mathbb{T}^{n}\right)$. (As a consequence of $\|\widehat{f}\|_{L^{\infty}} \leq\|f\|_{L^{1}}$.)

- $X=L^{p}\left(\mathbb{T}^{n}\right), 1<p \leq 2$. (Hausdorff-Young Inequality).

- $X=\Lambda^{s}\left(\mathbb{T}^{1}\right)=B_{\infty, \infty}^{s}(\mathbb{T}), 0<s<1$. In fact, by Lemma 3.5, if $p>\left(s+\frac{1}{2}\right)^{-1}$ then

$$
\|\widehat{f}\|_{L^{p}} \lesssim\|f\|_{\Lambda^{s}}
$$

Hence

$$
\sum_{\xi \in \mathbb{Z}^{n}}\left\|H_{\xi}\right\|_{B_{p_{2}, q_{2}}^{w_{2}}}^{r}\left\|G_{\xi}\right\|_{(X)^{\prime}}^{r} \lesssim \sum_{\xi \in \mathbb{Z}^{n}} C^{r}\langle\xi\rangle^{r\left(w_{2}+m+\delta(2 k)\right)}<\infty .
$$

As a consequence of this, we obtain the $r$-nuclearity of $T_{a}: X \rightarrow B_{p_{2}, q_{2}}^{w_{2}}$, where $0 \leq w_{2}<2 k-n, m<-\frac{n}{r}-w_{2}-\delta(2 k), 1 \leq p_{2} \leq \infty$ and $1 \leq q_{2} \leq \infty$. 
We end this section with the following result on $r$-nuclearity of periodic operators on Hölder spaces.

Corollary 3.7. Let $0<r \leq 1,0<s, w<1$, and $0 \leq \rho, \delta \leq 1$. Let us assume that $a \in S_{\rho, \delta, 0,2}^{m}(\mathbb{T} \times \mathbb{Z})$. If $m<-\frac{1}{r}-w-2 \delta$, then $T_{a}: \Lambda^{s}(\mathbb{T}) \rightarrow \Lambda^{w}(\mathbb{T})$ is a $r$-nuclear operator.

Proof. Let us apply Theorem 3.6 with $X^{(1)}=B_{\infty, \infty}^{s}(\mathbb{T})=\Lambda^{s}(\mathbb{T}), k=1$, and $w_{2}=w$.

\section{TRACE FORMUlaE FOR $r$-NUClEAR PSEUDO-DIFFERENTIAL OPERATORS} ON BESOV SPACES

In this section we provide trace formulae for $r$-nuclear operators on periodic Besov spaces. We recall the following result due to Grothendieck (see [22]).

Theorem 4.1. Let $E$ be a Banach space and $T: E \rightarrow E$ be a $\frac{2}{3}$-nuclear operator. Then the nuclear trace agrees with the sum of the eigenvalues $\lambda_{n}(T)$ of $T$, (with multiplicities counted).

Using this result we have our first Grothendieck-Lidskii trace formula for $r$ nuclear operators on periodic Besov spaces:

Theorem 4.2. Let $0<r \leq \frac{2}{3}, 0<\alpha \leq \frac{1}{2}, 0 \leq \rho, \delta \leq 1$, and $k>\frac{n}{2}, k \in \mathbb{N}$. Let us consider $a \in S_{\rho, \delta, 0,2 k}^{m}\left(\mathbb{T}^{n} \times \mathbb{Z}^{n}\right)$. Under the following conditions,

- $0<w_{1}=\alpha \cdot n<2 k-n, 1<p_{1} \leq 2, q_{1}=\left(\alpha+\frac{1}{p_{1}^{\prime}}\right)^{-1}$.

- $m<-\frac{n}{r}-w_{1}-\delta(2 k)$,

the pseudo-differential operator $T_{a}: B_{p_{1}, q_{1}}^{w_{1}} \rightarrow B_{p_{1}, q_{1}}^{w_{1}}$ is $r$-nuclear and the trace of $T$, is given by

$$
\operatorname{Tr}\left(T_{a}\right)=\sum_{\xi \in \mathbb{Z}^{n}} \int_{\mathbb{T}^{n}} a(x, \xi) d x=\sum_{n} \lambda_{n}\left(T_{a}\right)
$$

where $\lambda_{n}\left(T_{a}\right)$ is the sequence of eigenvalues of $T_{a}$ with multiplicities taken into account.

Proof. We observe that by Theorem 3.3, the operator $T_{a}$ is $r$-nuclear. Let us denote by $\lambda_{n}\left(T_{a}\right)$ the sequence of eigenvalues of $T_{a}$ with multiplicities taken into account. Since $0<r \leq \frac{2}{3}$, from the Theorem 4.1 we obtain

$$
\begin{aligned}
\sum_{n} \lambda_{n}\left(T_{a}\right)= & \operatorname{Tr}\left(T_{a}\right)=\sum_{\xi \in \mathbb{Z}^{n}} G_{\xi}\left(H_{\xi}\right) \\
& =\sum_{\xi \in \mathbb{Z}^{n}} \widehat{H}_{\xi}(\xi)=\sum_{\xi \in \mathbb{Z}^{n}} \widehat{a}(0, \xi)=\sum_{\xi \in \mathbb{Z}^{n}} \int_{\mathbb{T}^{n}} a(x, \xi) d x
\end{aligned}
$$

Corollary 4.3. Let $0<r \leq 1,0<\alpha \leq \frac{1}{2}, 0 \leq \rho, \delta \leq 1$, and $k>\frac{n}{2}, k \in \mathbb{N}$. Let us consider $a \in S_{\rho, \delta, 0,2 k}^{m}\left(\mathbb{T}^{n} \times \mathbb{Z}^{n}\right)$. Under the following conditions,

- $0<w_{1}=\alpha \cdot n<2 k-n, 1<p_{1} \leq 2, q_{1}=\left(\alpha+\frac{1}{p_{1}^{\prime}}\right)^{-1}$.

- $m<-\frac{n}{r}-w_{1}-\delta(2 k)$, 
the pseudo-differential operator $T_{a}: B_{p_{1}, q_{1}}^{w_{1}} \rightarrow B_{p_{1}, q_{1}}^{w_{1}}$ is $r$-nuclear and the trace of $T_{a}$, is given by

$$
\operatorname{Tr}\left(T_{a}\right)=\sum_{\xi \in \mathbb{Z}^{n}} \int_{\mathbb{T}^{n}} a(x, \xi) d x .
$$

Proof. By Theorem 3.3 $T_{a}$ is a $r$-nuclear operator. The trace formula (4.2) now follows from Theorem 2.2.

In order to prove our next trace formula, we use the following result by $\mathrm{O}$. Reinov and Q. Latif, which extends the Grothendieck-Lidskii trace formula for $r \in\left(\frac{2}{3}, 1\right]$ (see [36]).

Theorem 4.4. Let $Y$ be a subspace of an $L^{p}(\mu)$ space, $1 \leq p \leq \infty$. Assume that $T$ is a r-nuclear operator on $Y$, where $1 / r=1+|1 / 2-1 / p|$. Then, the (nuclear) trace of $T$ is well defined, the sequence of eigenvalues $\lambda_{n}(T)$ of $T$ (with multiplicities counted) is summable and

$$
\operatorname{Tr}(T)=\sum_{n} \lambda_{n}(T)
$$

Notice that as a consecuence of Theorem 3.3, if $a \in S_{\rho, \delta, 0,2 k}^{m}\left(\mathbb{T}^{n} \times \mathbb{Z}^{n}\right), r=1$, $1<p_{1}<2, \alpha=\frac{1}{p_{1}}-\frac{1}{2}$, and $w_{1}, m$ are index satisfying the conditions stated there, the pseudo-differential operator $T_{a}: B_{p_{1}, q_{1}}^{w_{1}}\left(\mathbb{T}^{n}\right) \rightarrow B_{p_{2}, q_{2}}^{w_{2}}\left(\mathbb{T}^{n}\right)$ is nuclear. It follows from Theorem 5.2 of [38] that $Y=B_{p_{1}, 2}^{\omega_{1}}$ is a subspace of $L^{2}\left(\mathbb{T}^{n}\right)$. By applying Theorem 4.4 (with $p=2, Y=B_{p_{1}, 2}^{\omega_{1}}$ and $r=1$ ), we conclude that (4.3) holds for $T=T_{a}$. From the Theorem 2.2, we have that

$$
\operatorname{Tr}\left(T_{a}\right)=\sum_{\xi \in \mathbb{Z}^{n}} \int_{\mathbb{T}^{n}} a(x, \xi) d x=\sum_{n} \lambda_{n}\left(T_{a}\right) .
$$

Thus, we summarize this facts in the following

Theorem 4.5. Let $1<p_{1}<2$ and $\alpha=\frac{1}{p_{1}}-\frac{1}{2}$. Let $0 \leq \rho, \delta \leq 1$, and $k>\frac{n}{2}$, $k \in \mathbb{N}$. Let us consider $a \in S_{\rho, \delta, 0,2 k}^{m}\left(\mathbb{T}^{n} \times \mathbb{Z}^{n}\right)$. Under the following conditions,

- $0<w_{1}=\alpha \cdot n<2 k-n$.

- $m<-n-w_{1}-\delta(2 k)$,

The operator $T_{a}$ is nuclear on $B_{p_{1}, 2}^{\omega_{1}}$ and

$$
\operatorname{Tr}\left(T_{a}\right)=\sum_{\xi \in \mathbb{Z}^{n}} \int_{\mathbb{T}^{n}} a(x, \xi) d x=\sum_{n} \lambda_{n}\left(T_{a}\right) .
$$

The sequence $\lambda_{n}\left(T_{a}\right)$ is conformed by the eigenvalues of $T_{a}$ with multiplicities counted.

\section{Trace formulae For Fourier multipliers on the torus}

In this section we provide trace formulae for $r$-nuclear Fourier multipliers on periodic Besov spaces. We denote by $S_{0}^{m}\left(\mathbb{T}^{n} \times \mathbb{Z}^{n}\right)$ the set of functions $a: \mathbb{Z}^{n} \rightarrow \mathbb{C}$ satisfying $|a(\xi)| \leq C\langle\xi\rangle^{m}$. 
Theorem 5.1. Let $0<r \leq \frac{2}{3}$, and let $0<\alpha \leq \frac{1}{2}$. Let us consider $a(\xi) \in$ $S_{0}^{m}\left(\mathbb{T}^{n} \times \mathbb{Z}^{n}\right)$. Under the following conditions,

- $1<p_{1} \leq 2, q_{1}=\left(\alpha+\frac{1}{p_{1}^{\prime}}\right)^{-1}$,

- $m<-\frac{n}{r}-\alpha \cdot n$,

the Fourier multiplier $T_{a}: B_{p_{1}, q_{1}}^{w} \rightarrow B_{p_{1}, q_{1}}^{w}$ is $r$-nuclear for every $w \in \mathbb{R}$ and the trace of $T$, is given by

$$
\operatorname{Tr}\left(T_{a}\right)=\sum_{\xi \in \mathbb{Z}^{n}} a(\xi)=\sum_{n} \lambda_{n}\left(T_{a}\right)
$$

where $\lambda_{n}\left(T_{a}\right)$ is the sequence of eigenvalues of $T_{a}$ with multiplicities taken into account.

Proof. We observe that by Theorem 3.3, the operator $T_{a}$ is $r$-nuclear on $B_{p_{1}, q_{1}}^{\alpha \cdot n}\left(\mathbb{T}^{n}\right)$ with $0<\alpha \cdot n<2 k-n$. By using Theorem 2.3 we extend this result to every $w \in \mathbb{R}$. Now, if we denote by $\lambda_{n}\left(T_{a}\right)$ the sequence of eigenvalues of $T_{a}$ with multiplicities taken into account and considering $0<r \leq \frac{2}{3}$, from the Theorem 4.1 we obtain

$$
\begin{aligned}
\sum_{n} \lambda_{n}\left(T_{a}\right)= & \operatorname{Tr}\left(T_{a}\right)=\sum_{\xi \in \mathbb{Z}^{n}} G_{\xi}\left(H_{\xi}\right) \\
& =\sum_{\xi \in \mathbb{Z}^{n}} \widehat{H}_{\xi}(\xi)=\sum_{\xi \in \mathbb{Z}^{n}} \widehat{a}(0, \xi)=\sum_{\xi \in \mathbb{Z}^{n}} a(\xi) .
\end{aligned}
$$

An immediate consequence of the Theorem above is the following.

Corollary 5.2. Let $0<r \leq 1$, and let $0<\alpha \leq \frac{1}{2}$. Let us consider $a(\xi) \in$ $S_{0}^{m}\left(\mathbb{T}^{n} \times \mathbb{Z}^{n}\right)$. Under the following conditions,

- $1<p_{1} \leq 2, q_{1}=\left(\alpha+\frac{1}{p_{1}^{\prime}}\right)^{-1}$.

- $m<-\frac{n}{r}-\alpha \cdot n$,

the Fourier multiplier $T_{a}: B_{p_{1}, q_{1}}^{w} \rightarrow B_{p_{1}, q_{1}}^{w}$ is $r$-nuclear for every $w \in \mathbb{R}$ and the nuclear trace of $T_{a}$, is given by

$$
\operatorname{Tr}\left(T_{a}\right)=\sum_{\xi \in \mathbb{Z}^{n}} a(\xi)
$$

Proof. By Theorem 3.3 $T_{a}$ is a $r$-nuclear operator. The trace formula (4.2) now follows from Theorem 2.2.

Now we present the following result which can be proved by using similar arguments as above.

Theorem 5.3. Let $1<p_{1}<2$ and $\alpha=\frac{1}{p_{1}}-\frac{1}{2}$. Let us consider $a(\xi) \in S_{0}^{m}\left(\mathbb{T}^{n} \times\right.$ $\left.\mathbb{Z}^{n}\right)$. If $m<-n-\alpha \cdot n$, the Fourier multiplier $T_{a}$ is a nuclear operator on $B_{p_{1}, 2}^{w}$ for every $w \in \mathbb{R}$ and

$$
\operatorname{Tr}\left(T_{a}\right)=\sum_{\xi \in \mathbb{Z}^{n}} a(\xi)=\sum_{n} \lambda_{n}\left(T_{a}\right)
$$


The sequence $\lambda_{n}\left(T_{a}\right)$ is conformed by the eigenvalues of $T_{a}$ with multiplicities counted.

Remark 5.4. Now, we discuss our main results in the periodic case. Theorem 3.3, if we consider smooth symbols (i.e with derivatives of arbitrary order), we obtain the $r$-nuclearity in Besov spaces of pseudo-differential on the torus $\mathbb{T}^{n}$, associated to symbols of order less that $-\frac{n}{r}$, and some conditions of the parameters $p_{i}, q_{i}$ and on $w_{i}$. This is a expected fact, in analogy with some results by Ruzhansky and Delgado in $L^{p}$ spaces (c.f. $[11,12,13,14]$ ). The conclusion above is same for Theorem 3.4. Also, it is important to mention that trace formulae obtained in the last two sections are versions in Besov spaces of ones obtained by Delgado and Wong in $L^{p}$ spaces [9].

We end this section with the following examples where, by using results above we compute the trace of the heat kernel and suitable powers of the Bessel potential on periodic Besov spaces.

Example 5.5. Let $\mathcal{L}_{\mathbb{T}^{n}}$ be the Laplacian on the torus $\mathbb{T}^{n}$, for every $s \in \mathbb{R}$, the Bessel potential of order $s$ denoted by $\left(I-\mathcal{L}_{\mathbb{T}^{n}}\right)^{s}$ is the periodic operator with symbol $a_{s}(x, \xi)=\langle\xi\rangle^{s}$. If $0<r \leq 1$, and $0<\alpha \leq \frac{1}{2}$, by using Corollary 4.3, under the following conditions,

$$
\text { - } \alpha>0,1<p_{1} \leq 2, q_{1}=\left(\alpha+\frac{1}{p_{1}^{\prime}}\right)^{-1} \text {, and } m<-\frac{n}{r}-\alpha \cdot n \text {, }
$$

the operator $\left(I-\mathcal{L}_{\mathbb{T}^{n}}\right)^{-\frac{m}{2}}$ with symbol $a(\xi)=\langle\xi\rangle^{-m} \in S_{1,0}^{-m}\left(\mathbb{T}^{n} \times \mathbb{Z}^{n}\right)$ is $r$-nuclear on every $B_{p_{1}, q_{1}}^{w_{1}}$ and its trace is given by

$$
\operatorname{Tr}\left(\left(I-\mathcal{L}_{\mathbb{T}^{n}}\right)^{-\frac{m}{2}}\right)=\sum_{\xi \in \mathbb{Z}^{n}}\langle\xi\rangle^{-m} .
$$

Example 5.6. If $t>0$, the heat kernel $e^{-t \mathcal{L}_{\mathbb{T}^{n}}}$ is the operator with symbol $a_{t}(x, \xi)=e^{-t|\xi|^{2}} \in S_{1,0}^{-\infty}\left(\mathbb{T}^{n} \times \mathbb{Z}^{n}\right)$. Newly, by Corollary 4.3 , if $w_{1}, p_{1}$ and $q_{1}$ satisfy the condition above, $e^{-t \mathcal{L}_{\mathbb{T}^{n}}}$ is a $r$-nuclear operator on $B_{p_{1}, q_{1}}^{w_{1}}$ and its trace is given by

$$
\operatorname{Tr}\left(e^{-t \mathcal{L}_{\mathbb{T}^{n}}}\right)=\sum_{\xi \in \mathbb{Z}^{n}} e^{-t|\xi|^{2}}
$$

Acknowledgments. The author is indebted with Alexander Cardona for helpful comments on an earlier draft of this paper. The author would like to warmly thank the anonymous referee for his remarks and important advices leading to several improvements of the original paper.

\section{REFERENCES}

1. Achour, D. Rueda, P. Sánchez-Pérez, E. A. Yahi, R. Lipschitz operator ideals and the approximation property. J. Math. Anal. Appl. 436 (2016), no. 1, 217-236.

2. Alberti, G. Csönyei, M. Pelczyński, A. Preiss, D. BV has the bounded approximation property. J. Geom. Anal. 15 (2005), no. 1, 1-7.

3. Böttcher, A. Grudsky, S. Huybrechs, D. Iserles, A. First-order trace formulae for the iterates of the Fox-Li operator. A panorama of modern operator theory and related topics, 207-224, Oper. Theory Adv. Appl., 218, Birkhuser Springer Basel AG, Basel, 2012. 
4. Anisca, R; Chlebovec, C. Subspaces of $l^{2}(X)$ without the approximation property. J. Math. Anal. Appl. 395 (2012), no. 2, 523-530.

5. Bloom, W. R. Bernstein's inequality for locally compact Abelian groups. Journal the Australian mathematical society 17 (1974), 88-101.

6. Brudnyi, A. On the approximation property for Banach spaces predual to H-spaces. J. Funct. Anal. 263 (2012), no. 9, 2863-2875.

7. Cardona, D. On the singular values of the Fox-Li operator. J. Pseudo-Differ. Oper. Appl. 6 (2015), no. 4, 427-438.

8. Cardona, D. Besov continuity for Multipliers defined on compact Lie groups, Palest. J. Math. Vol. 5(2) 35-44 (2016).

9. Delgado, J., Wong, M.W.: $L^{p}$-nuclear pseudo-differential operators on $\mathbb{Z}$ and $\mathbb{S}^{1}$., Proc. Amer. Math. Soc., 141 (2013) no. 11, 3935-3942.

10. Delgado, J.: Trace formulas for nuclear operators in spaces of Bochner integrable functions., Monatsh. Math, 172 (2013) (3-4) 259-275.

11. Delgado, J. Ruzhansky, M.: $L^{p}$-nuclearity, traces, and Grothendieck-Lidskii formula on compact Lie groups., J. Math. Pures Appl. (9) 102 (2014), no. 1, 153-172.

12. Delgado, J. Ruzhansky,M.: Schatten classes and traces on compact groups, Math. Res. Lett., to appear, arXiv:1303.3914.

13. Delgado, J. Ruzhansky, M.: Schatten classes on compact manifolds: kernel conditions. J. Funct. Anal. 267 (2014), no. 3, 772-798.

14. Delgado, J. Ruzhansky, M.: Kernel and symbol criteria for Schatten classes and r-nuclearity on compact manifolds. C. R. Math. Acad. Sci. Paris 352 (2014), no. 10, 779-784.

15. Delgado, J.: On the $r$-nuclearity of some integral operators on Lebesgue spaces. Tohoku Math. J. (2) 67 (2015), no. 1, 125-135.

16. Delgado, J. Ruzhansky, M. The metric approximation property of variable Lebesgue spaces and nuclearity. arXiv:1410.4687.

17. Delgado, J. Ruzhansky, M. Wang, B. Approximation property and nuclearity on mixednorm $L^{p}$, modulation and Wiener amalgam spaces. J. Lond. Math. Soc., 94 (2016), 391-408.

18. Delgado, J. Ruzhansky, M. Wang, B. Grothendieck-Lidskii trace formula for mixed-norm $L^{p}$ and variable Lebesgue spaces. to appear in J. Spectr. Theory.

19. Delgado, J. Ruzhansky, M. Tokmagambetov, N. Schatten classes, nuclearity and nonharmonic analysis on compact manifolds with boundary, arXiv:1505.02261, to appear in J. Math. Pures Appl.

20. Enflo, P. A counterexample to the approximation problem in Banach spaces. Acta Math. 130 (1973), 309-317.

21. Feichtinger, H. Führ, H. Pesenson, I. Geometric Space-Frequency Analysis on Manifolds, arXiv:1512.08668.

22. Grothendieck, A.: Produits tensoriels topologiques et espaces nucléaires, Memoirs Amer. Math. Soc. 16, Providence, 1955 (Thesis, Nancy, 1953).

23. Haagerup, U; de Laat, T. Simple Lie groups without the approximation property. Duke Math. J. 162 (2013), no. 5, 925-964.

24. Hermann, K. Eigenvalue distribution of compact operators. Operator Theory: Advances and Applications, 16. Birkhuser Verlag, Basel, 1986.

25. Kim, J. M; Lee, K. Y. Weak approximation properties of subspaces. Banach J. Math. Anal. 9 (2015), no. 2, 248-252.

26. Kim, J. M; Zheng, B. The strong approximation property and the weak bounded approximation property. J. Funct. Anal. 266 (2014), no. 8, 5439-5447.

27. Lancien, G.; Perneck, E. Approximation properties and Schauder decompositions in Lipschitz-free spaces. J. Funct. Anal. 264 (2013), no. 10, 2323-2334.

28. Lee, K. Y. The separable weak bounded approximation property. Bull. Korean Math. Soc. 52 (2015), no. 1, 69-83.

29. Lee, K. Y. Approximation properties in fuzzy normed spaces. Fuzzy Sets and Systems 282 (2016), 115-130. 
30. Li, J; Fang, X. C p-weak approximation property in Banach spaces. (Chinese) Chinese Ann. Math. Ser. A 36 (2015), no. 3, 247-256.

31. Lima, A. Lima, V. Oja, E. Bounded approximation properties in terms of $C[0,1]$. Math. Scand. 110 (2012), no. 1, 45-58.

32. Mayer, D. H. On the thermodynamic formalism for the Gauss map. Comm. Math. Phys. 130 (1990), no. 2, 311-333.

33. Oja, E; Veidenberg, S. Lifting convex approximation properties from Banach spaces to their dual spaces and the related local reflexivity. J. Math. Anal. Appl. 436 (2016), no. 2, 729-739

34. Pietsch, A. Operator ideals. Mathematische Monographien, 16. VEB Deutscher Verlag der Wissenschaften, Berlin, 1978.

35. Pietsch, A. History of Banach spaces and linear operators. Birkhäuser Boston, Inc., Boston, MA, 2007.

36. Reinov, O.I., Laif, Q., Grothendieck-Lidskii theorem for subspaces of Lpspaces. Math. Nachr., Volume 286, Issue 2-3, 279-282, (2013).

37. Roginskaya, M. Wojciechowski, M. Bounded Approximation Property for Sobolev spaces on simply-connected planar domains. arXiv:1401.7131

38. Nursultanov, E. Ruzhansky, M. Tikhonov, S. Nikolskii inequality and Besov, TriebelLizorkin, Wiener and Beurling spaces on compact homogeneous manifolds. Ann. Sc. Norm. Super. Pisa Cl. Sci., Vol. XVI, (2016), 981-1017.

39. Fischer V., Ruzhansky M., Quantization on nilpotent Lie groups, Progress in Mathematics, Vol. 314, Birkhauser, 2016.

40. Ruzhansky, M., Turunen, V.:Pseudo-differential Operators and Symmetries: Background Analysis and Advanced Topics Birkhaüser-Verlag, Basel, (2010)

41. Ruzhansky M., Turunen V., Global quantization of pseudo-differential operators on compact Lie groups, SU(2) and 3-sphere, Int. Math. Res. Not. IMRN 2013, no. 11, 2439-2496.

42. Szász, O. Über den Konvergenzexponenten der Fouriersohen Reihen gewisser Funktionenklassen, Sitzungsberichte der Bayerischen Akademie der Wissenschaften Mathematischphysikalische Klasse. 135-150, (1922).

43. Szász, O. Über die Fourierschen gewiser Funktionenklassen. Mathematische Annalen. 530536. (1928)

Duván CARDONA:

Department of Mathematics

UNIVERSIDAD DE LOS ANDES

BogOTÁ

Colombia

E-mail address d.cardona@uniandes.edu.co; duvanc306@gmail.com 